\title{
Role of Assistive Devices on Gait in Patients with Incomplete Spinal Cord Injury: Systematic Review
}

\author{
Wessam A. Alrefaie ${ }^{* 1}$, Nahed A. Salem², Mahmoud S. El fakharany ${ }^{3}$, Mahmoud Y. El Zanaty ${ }^{2}$ \\ ${ }^{1}$ Department Physiotherapist at Police Hospital Authority, Cairo, Egypt \\ ${ }^{2}$ Department of Physical Therapy for Neuromuscular Disorder, ${ }^{3}$ Department of Physical Therapy for Pediatrics, \\ Faculty of Physical Therapy, Cairo University, Cairo, Egypt \\ *Corresponding author: Wessam A. Alrefaie, Mobile: (+20)01063045651, E-Mail: wessamrefaay1991@gmail.com
}

\begin{abstract}
Background: People with incomplete spinal cord injury disabilities can be able to live a healthy, productive, and dignified life by using Assistive devices as their role in improving gait. Facilitate locomotion rehabilitation. And enable people with incomplete SCI to ambulate in an upright position. Objective: This systematic review aimed to examine the effectiveness of the role of using assistive devices in gait rehabilitation in patients with incomplete SCI.

Material and Methods: Studies were identified from 2000 to 2020 by electronic search using PubMed, Cochrane Database of Systematic Reviews, Google Scholar, and Physiotherapy Evidence Database (Pedro). They were reviewed if they were randomized control trials focused on the effectiveness of Assistive Devices on Gait in Patients in age more than 18 years with incomplete Spinal Cord Injury being published in English. Eight studies were selected according to inclusive and exclusive criteria and descriptive analysis was conducted due to heterogeneity. Results: Eight trials were identified with good quality methodology. Descriptive analysis was applied for three studies that supported the use of assistive devices for those patients and meta-analysis was applied for five studies. The mean difference across all the five studies is -0.69 (95\% CI -0.93, -0.45). According to AACPDM, there is level II evidence that supports the use of the assistive device as a method to be able to live a healthy, productive, and dignified life. Conclusion: The current level of evidence supports the effectiveness of assistive devices in improving gait in patients with incomplete spinal cord injury.
\end{abstract}

Keywords: Incomplete Spinal cord injury, Types of incomplete SCI, Orthoses, Lower limbs disabilities, Types of orthoses.

\section{INTRODUCTION}

Spinal cord injury is any damage to the spinal cord which blocks communication between the brain and the body. A person's motor, sensory, and reflex messages are affected and may be unable to get past the damage in the spinal cord ${ }^{(\mathbf{1})}$. Incomplete Lesion: nerves slightly damaged. Recovery is possible, but never to pre-injury level ${ }^{(\mathbf{1})}$. The clinical outcomes of incomplete SCI depend on the severity and location of the lesion ${ }^{(2)}$. According to the National Spinal Cord Injury Statistical Center, _282,000 persons are living with spinal cord injury (SCI) in the United States, and 17,000 new SCI cases occur each year. More than $90 \%$ of SCI cases are traumatic and caused by incidences such as traffic accidents, violence, sports, or falls. The Male: female ratio of 2:1 for SCI, which occurs frequently in adults compared to children. Demographically, men are mostly affected during their early and late adulthood ( $3^{\text {rd }}$ and $8^{\text {th }}$ decades of life) Difficulty walking is very common following a spinal cord injury (SCI) ${ }^{(\mathbf{1})}$.

People with an "incomplete" SCI have more potential to regain walking than those with a "complete" SCI which depends on many factors including Level of injury, severity of the injury, level of sensation, level of pain, time since injury, age, and level of fitness ${ }^{(3)}$. Other Related Problems such as Spasticity and Joint Problems e.g., Contractures It is difficult to predict if a person with SCI will regain walking abilities
(4). The proportion of patients with SCI who walked at inpatient discharge with devices/braces and without physical assistance: ASIA A (motor and sensory complete): $6.4 \%$. ASIA B (motor complete, sensory incomplete): $23.5 \%$. ASIA C (motor and sensory incomplete, generally weaker legs): $51.4 \%$. ASIA D (motor and sensory incomplete, generally stronger legs): $88.9 \%^{(5,6,7)}$.

Devices have been developed to assist patients suffering from SCI with mobility and to facilitate locomotion rehabilitation ${ }^{(8)}$. There has been an evolution in gait rehabilitation programs for people with ISCI in recent decades, from manually assisted over-ground training; body-weight-supported treadmill training (BWSTT) to robotic-assisted gait training (RAGT) ${ }^{(\mathbf{9}, 10)}$, which is a powered exoskeleton. Powered exoskeletons are motorized orthoses placed over a person's limb with joint parts corresponding to those of the human body. Their purpose is to facilitate standing and walking, as well as assist in rehabilitation ${ }^{(\mathbf{1 1})}$.

The RAGT focuses on the correct performance of gait movements. Therapy is performed at low speed and the level of assistance by the system can be adjusted based on the patient's ability to step ${ }^{(12)}$. Body weightsupported treadmill training (BWSTT) can enhance locomotor activity after a spinal cord injury. In this approach, partial body weight support is provided by an overhead harness while leg movements are assisted by therapists and a moving treadmill belt ${ }^{(13,14)}$. There are 
also other rehabilitation approaches, such as functional electrical stimulation or bracing, that enable a person to stand up and practice overground walking ${ }^{(15)}$. Functional electrical stimulation (FES), which has a long tradition as an orthotic and therapeutic aid in the rehabilitation of walking after paraparesis. Direct stimulation of motor neurons, artificial activation of spinal neural circuits, and stimulation of dermatomes have been employed successfully to augment artificially the movement of the affected lower extremity, usually during the swing phase (15). There are other assistive devices (wheelchairs, canes, crutches, walkers or walking frames, walking sticks, tricycles, canes, parallel bars, treadmills, braces, or orthoses ${ }^{(16)}$.

All these devices are designed to solve the problem of standing and walking, but there are some other important notes, which should be considered. For example, the size and weight. Systematic reviews and meta-analysis lie on top of the evidence pyramid both in public health and clinical medicine ${ }^{\left({ }^{(17)}\right.}$. Decision-making is the process by which evidence is (or is not) applied to practice. The statement "evidence alone does not make decisions, people do" reflects the integral role of the therapist in the translation of evidence to practice. Therapists make decisions on complex issues related to examination, prognosis, expected outcomes, the plan of care, and the coordination of care daily ${ }^{(\mathbf{1 8})}$.

This systematic review aimed to examine the effectiveness of the role of using assistive devices in gait rehabilitation in patients with incomplete SCI.

\section{Methods:}

A systematic search was conducted for best research evidence for the effectiveness of assistive devices on gait in SCI. Using electronic databases (PubMed, Cochrane (CENTRAL), PEDro, EKB databases).

Eligibility criteria: Studies were selected according to the criteria outlined below.

- Types of studies: Published full-text randomized trials.

- Types of participants: Trials included enrolled adults (age $>18$ years) of both sex with incomplete spinal cord injury.

- Types of Intervention: Assistive devices

- Control/Comparator: Any comparator is included. Comparisons of interventions with control, placebo, or standard care

- Outcomes: Anyone/all/some of these criteria:

The primary outcome measures used:

- Walking Index for Spinal Cord Injury (WISCI).

- $\quad 10$-meter walk test (10MWT).

- 6MWT.

- The Functional Independence Measure (FIM).

Secondary investigated outcomes were:

- Achieve maximum independence.

- Increase sense of control.

- Increase participation in life roles.
- Increases efficiency.

- Provides a level of privacy and dignity.

- Decreases caregiver burnout.

- Supports function in a variety of environments

Search strategy:

Search methods for identification of studies:

1- Searching electronic databases: The following sources will be searched from 2010 to 2020 :

- Google scholar.

- PubMed.

- The Cochrane Central register of controlled trials

- Physiotherapy Evidence Database (PEDro).

2- Hand searching.

3- Searching other resources: To identify other relevant trial data:

- Reference lists of review articles and primary trials found will be screened

\section{Keywords:}

The following keywords are used in the search strategy. "assistive devices" or "robotic-assisted gait training" or "RAGT" or robotic-assisted locomotor training" or "Exoskeleton-assisted gait training" or" Lokomat Robotic-Assisted " or "Body-WeightSupported Training "or "BWS" or "functional electrical stimulation " or "FES" or "hydrotherapy" or "aquatic therapy" or "underwater exercise" or " orthoses" AND "walking" or "gait" or "locomotion" AND "incomplete spinal cord injury" or" spinal cord injury "or "ISCI" or "SCI".

\section{Selection of studies:}

Three review authors will independently scan the abstract, title, or both, of every record retrieved, to determine which studies should be assessed further. All potentially relevant articles as the full text will be investigated.

\section{Exclusion criteria:}

1- The studies were designed other than randomized trials (e.g. a case report, case series, and review articles). 2- Articles published in non- English language and not published.

3-Studies assessed complete spinal cord injury or incomplete SCI with other interventions (Combined interventions).

4- Patient targeted (age younger than 18 years old).

5- Populations other than (ISCI).

\section{Data extraction and management:}

Studies that fulfill inclusion criteria, two review authors were independently abstract key participant, intervention characteristics, report data on efficacy outcomes and adverse events using standard data extraction templates, with any disagreements to be resolved by discussion, or if required by a third author. Data will be extracted and documented in data extraction form: Study design, Objectives, Inclusion and exclusion criteria, Participant's characteristics, Sample size and 
distribution, Interventions, comparisons, treatment dosages, outcomes assessed, and results ${ }^{(19)}$.

Methodological Quality Assessment of the Studies:

The methodological quality assessment will be carried out by two reviewers using Physical Therapy Evidence Database (PEDRo) scale which independently assesses studies against 10 criteria (e.g.: eligibility criteria, randomization, blinding, allocation concealment, and other aspects) and categorizes them by score as follows: excellent (9-10), good (6-8), fair (4$5)$, and poor $(<4)$. If there is some sort of disagreement, discussion, and referral to another reviewer to reach consensus will be considered ${ }^{(\mathbf{2 0})}$.
Search Results:

One hundred thirty-seven studies are the number of total studies identified from the initial electronic search in PubMed, CDSR, Goggle Scholar, and PEDRo as well as manual Search from January 2010 to 2020. Ten studies were removed Because of duplication. Twenty-nine studies were excluded after screening the title and the Abstract and five studies were excluded after full-text screening as they were not RCTs.

The remaining eight studies were included. Descriptive analysis was performed for three studies and meta-analysis was performed for five studies.

Table (1): RCT Studies Included In the Study.

\begin{tabular}{|c|c|c|}
\hline No. & Authors (Year) & Title \\
\hline 1 & $\begin{array}{l}\text { Labruyère et al. } \\
\text { (2014) }\end{array}$ & $\begin{array}{l}\text { Strength training versus robot-assisted gait training after incomplete } \\
\text { spinal cord injury: A randomized pilot study in patients depending on } \\
\text { walking assistance }\end{array}$ \\
\hline 2 & Piira et al. (2019) ${ }^{(22)}$ & $\begin{array}{l}\text { Robot-Assisted Locomotor Training Did Not Improve Walking } \\
\text { Function In Patients With Chronic Incomplete Spinal Cord Injury: A } \\
\text { Randomized Clinical Trial }\end{array}$ \\
\hline 3 & $\begin{array}{l}\text { Alcobendas et al. } \\
\text { (2012) }\end{array}$ & $\begin{array}{l}\text { Lokomat robotic-assisted versus overground training within } 3 \text { to } 6 \\
\text { months of incomplete spinal cord lesion: Randomized } \\
\text { controlled trial }\end{array}$ \\
\hline 4 & Chang et al. (2018) ${ }^{(24)}$ & $\begin{array}{l}\text { Exoskeleton-Assisted gait training to improve gait in individuals with } \\
\text { spinal cord injury: A pilot randomized study }\end{array}$ \\
\hline 5 & Wu et al. (2012) & $\begin{array}{l}\text { Robotic resistance treadmill training improves locomotor } \\
\text { function in human spinal cord injury: A pilot study }\end{array}$ \\
\hline 6 & Piira et al. (2019) ${ }^{(26)}$ & $\begin{array}{l}\text { Manually assisted body-weight supported locomotor training does not } \\
\text { re-establish walking in non-walking subject with chronic incomplete } \\
\text { spinal cord injury: A randomized clinical trial }\end{array}$ \\
\hline 7 & $\begin{array}{l}\text { Alexeeva } \text { et al. (2011) } \\
\text { (27) }\end{array}$ & $\begin{array}{l}\text { Comparison of training methods to improve walking in persons with } \\
\text { chronic spinal cord injury: a randomized clinical trial }\end{array}$ \\
\hline 8 & $\begin{array}{l}\text { Sadeghi } \text { et al. (2015) } \\
(28)\end{array}$ & $\begin{array}{l}\text { The effect of body-weight-supported training exercises } \\
\text { on functional ambulation profile in patients with paraplegic spinal } \\
\text { cord injury }\end{array}$ \\
\hline
\end{tabular}

Table (2): Methodology Assessment of The Reviewed Studies According to The PEDRo scale.

\begin{tabular}{|l|c|c|c|c|c|c|c|c|}
\hline \multicolumn{1}{|c|}{ Criteria } & $\begin{array}{c}\text { Labruyère } \\
\text { et al. } \\
(\mathbf{2 0 1 4})\end{array}$ & $\begin{array}{c}\text { Piira } \\
\text { et al. } \\
(\mathbf{2 0 1 9})\end{array}$ & $\begin{array}{c}\text { Alcobendas } \\
\text { et al. } \\
(\mathbf{2 0 1 2})\end{array}$ & $\begin{array}{c}\text { Chang } \\
\text { et al. } \\
(\mathbf{2 0 1 8}\end{array}$ & $\begin{array}{c}\text { Wu } \\
\text { et al. } \\
(\mathbf{2 0 1 2})\end{array}$ & $\begin{array}{c}\text { Piira } \\
\text { et al. } \\
(\mathbf{2 0 1 9})\end{array}$ & $\begin{array}{c}\text { Alexeeva } \\
\text { et al. } \\
(\mathbf{2 0 1 1})\end{array}$ & $\begin{array}{c}\text { Sadeghi } \\
\text { et al. } \\
(\mathbf{2 0 1 5})\end{array}$ \\
\hline 1. Specified eligibility criteria & YES & YES & YES & YES & YES & YES & YES & YES \\
\hline $\begin{array}{l}\text { 2. Random allocation of } \\
\text { Participants }\end{array}$ & YES & YES & YES & YES & YES & YES & YES & YES \\
\hline 3. Concealed allocation & YES & YES & YES & YES & NO & YES & YES & NO \\
\hline 4. Similar prognosis at baseline & YES & YES & YES & YES & YES & YES & YES & YES \\
\hline 5. Blinded participant & NO & NO & NO & NO & NO & NO & YES & NO \\
\hline 6. Blinded therapists & NO & NO & NO & NO & NO & NO & YES & NO \\
\hline 7. Blinded assessors & YES & YES & YES & NO & NO & YES & NO & YES \\
\hline $\begin{array}{l}\text { 8. More than 85\% follow-up for } \\
\text { at least one key outcome }\end{array}$ & YES & NO & YES & YES & NO & YES & YES & YES \\
\hline 9. 'Intent ion to treat ' analysis & YES & NO & YES & YES & NO & NO & YES & YES \\
\hline $\begin{array}{l}\text { 10. Between group statistical } \\
\text { analysis for at least one key outcome }\end{array}$ & YES & YES & YES & YES & YES & YES & YES & YES \\
\hline $\begin{array}{l}\text { 11. Point estimates of } \\
\text { variability for at least one key outcome }\end{array}$ & YES & YES & YES & YES & YES & YES & YES & YES \\
\hline PEDro score & $8 / 10$ & $6 / 10$ & $8 / 10$ & $7 / 10$ & $4 / 10$ & $7 / 10$ & $9 / 10$ & $7 / 10$ \\
\hline
\end{tabular}


The PEDro scale considers two aspects of trial quality, namely the "believability" (or "internal validity") of the trial and whether the trial contains sufficient statistical information to make it interpretable. It does not rate the "meaningfulness" (or "generalisability" or "external validity") of the trial or the size of the treatment effect. The first item on the PEDro scale (the item on eligibility criteria) is related to external validity, so it does not reflect the dimensions of quality assessed by the PEDro scale.

This item is not used to calculate the method score (which is why the 11-item scale gives a score out of 10). According to the PEDro guidelines, a positive answer to each of the criteria 2 to 11 will yield one point, obtaining a PEDro score between 0 to $10^{(29)}$.

Papers that had a PEDro score of seven or higher would be considered of 'high quality' while those with a PEDro score of five or six would be considered of moderate quality and those with a PEDro score of four or less would be considered of 'poor quality.

\section{Data synthesis and statistical analysis:}

This meta-analysis combined data at the study level. The outcome variables Ten-minute walking test (10 MWT), Six-meter walking test (6 MWT), lower extremity motor score- (LEMS), and Berg balance scale (BBS) are assessed as at the end of the intervention period as measured to improve walking function in patients with chronic incomplete spinal cord injury.

To allow the comparison of data from different scales, pooled statistics were calculated using standardized mean differences (SMDs), which were computed using the Review Manager program (RevMan software, version 5).
Means, mean change, and standard deviations (SDs) for the experimental group and control group were used to compute SMDs. The forest plots were computed by means, SD, and sample size effect for the control group versus experimental group for each study enters in meta-analysis.

If appropriate, the estimated effect size was calculated if the outcome variable was reported in $\geq 2$ studies and action research walking function score outcomes are continuous variables. Outcome variables were pooled across studies and analyzed using a random-effects model for data collated from all eligible acute studies obtained from review and data collated from all eligible intervention studies obtained from a review.

Action research walking function score outcomes measures with $95 \%$ confidence intervals were used to investigate differences. Heterogeneity measures the variability between studies using the $\mathrm{I}^{2}$ statistic to quantify the proportion of the total outcome attributed to variability among studies. Study variability and heterogeneity were tested by random-effects model and $\mathrm{I}^{2}$ statistic ${ }^{(30)}$.

The following values were used: $\mathrm{I}^{2}=0 \%-30 \%$ (no heterogeneity); $\mathrm{I}^{2}=30 \%-49 \%$ (moderate heterogeneity); $\mathrm{I}^{2}=50 \%-74 \%$ (substantial heterogeneity); and $\mathrm{I}^{2}=75 \%$ $100 \%$ (considerable heterogeneity). The statistical analysis was conducted by using the Review Manager Program for windows (RevMan software, version 5.4.1).

\section{RESULTS}

Study selection was presented in (Table 1).

- Study characteristics:

Study characteristics are presented in (Table 3). 
Table (3): Study characteristics

\begin{tabular}{|c|c|c|c|c|c|c|c|c|}
\hline $\begin{array}{c}\text { Authors } \\
\text { (year) }\end{array}$ & $\begin{array}{l}\text { Labruyèr } \\
\text { e et al. } \\
\text { (2014) }\end{array}$ & $\begin{array}{c}\text { Piira } \\
\text { et al. } \\
(2019) \\
\end{array}$ & $\begin{array}{c}\text { Alcobenda } \\
\text { s et al. } \\
(2012) \\
\end{array}$ & $\begin{array}{c}\text { Chang et } \\
\text { al. } \\
(2018) \\
\end{array}$ & $\begin{array}{c}\text { Wu } \\
\text { et al. } \\
(2012) .\end{array}$ & $\begin{array}{c}\text { Piira } \\
\text { et al. } \\
(2019) \\
\end{array}$ & $\begin{array}{c}\text { Alexeeva1 } \\
\text { et al. } \\
(2011) \\
\end{array}$ & $\begin{array}{l}\text { Sadeghi } \\
\text { et al. } \\
(2015)\end{array}$ \\
\hline Intervention & $\begin{array}{l}\text { - strength } \\
\text { Training } \\
\\
\text { - Robotic- } \\
\text { assisted } \\
\text { gait } \\
\text { training }\end{array}$ & $\begin{array}{l}\text { Convention } \\
\text { al physical } \\
\text { therapy } \\
\text { BWS on a } \\
\text { treadmill } \\
\text { then } \\
\text { Robotic- } \\
\text { Assisted } \\
\text { locomotor } \\
\text { Training }\end{array}$ & $\begin{array}{l}\text { Convention } \\
\text { al physical } \\
\text { therapy } \\
\text { Lokomat } \\
\text { Robotic- } \\
\text { Assisted }\end{array}$ & $\begin{array}{l}\text { Convention } \\
\text { al physical } \\
\text { therapy } \\
(\mathrm{CPT}) \text {. } \\
\text { exoskeleton } \\
\text {-assisted } \\
\text { gait training } \\
\text { (EGT) }\end{array}$ & $\begin{array}{l}\text { Robotic- } \\
\text { assisted } \\
\text { gait } \\
\text { training } \\
\text { Robotic } \\
\text { resisted } \\
\text { gait } \\
\text { training }\end{array}$ & $\begin{array}{l}\text { Convention } \\
\text { al physical } \\
\text { therapy } \\
\text { Manual } \\
\text { assisted } \\
\text { BWS } \\
\text { _loco motor } \\
\text { training }\end{array}$ & $\begin{array}{l}\text { Convention } \\
\text { al physical } \\
\text { therapy } \\
\text { (BWS) } \\
\text { ambulation } \\
\text { on a fixed } \\
\text { track and } \\
\text { treadmill }\end{array}$ & $\begin{array}{l}\text { Convention } \\
\text { al physical } \\
\text { therapy } \\
\text { Body- } \\
\text { Weight- } \\
\text { Supported } \\
\text { Treadmill } \\
\text { Training }\end{array}$ \\
\hline $\begin{array}{l}\text { Control } \\
\text { intervention }\end{array}$ & $\begin{array}{l}\text { Overgroun } \\
\text { d strength } \\
\text { training }\end{array}$ & $\begin{array}{l}\text { low- } \\
\text { intensity } \\
\text { usual care } \\
\text { from } \\
\text { their local } \\
\text { physical } \\
\text { therapist }\end{array}$ & $\begin{array}{l}\text { Traditional } \\
\text { overground } \\
\text { physical } \\
\text { therapy }\end{array}$ & $\begin{array}{l}\text { convention } \\
\text { al physical } \\
\text { therapy }\end{array}$ & $\begin{array}{l}\text { Robotic- } \\
\text { assisted } \\
\text { gait } \\
\text { training }\end{array}$ & $\begin{array}{l}\text { Traditional } \\
\text { physical } \\
\text { therapy }\end{array}$ & $\begin{array}{l}\text { Traditional } \\
\text { physical } \\
\text { therapy }\end{array}$ & $\begin{array}{l}\text { traditional } \\
\text { overground } \\
\text { training }\end{array}$ \\
\hline \multirow{4}{*}{$\begin{array}{l}\text { Outcome } \\
\text { Measures }\end{array}$} & Primary & Primary & Primary & Primary & Primary & Primary: & Primary & Primary \\
\hline & $\begin{array}{l}\text {-walking } \\
\text { speed } \\
\text {-Strength of } \\
\text { lower limbs } \\
\text {-balance }\end{array}$ & $\begin{array}{l}\text { Walking } \\
\text { speed } \\
\text { Strength of } \\
\text { Lower limbs } \\
\text {-balance } \\
\text {-Endurance }\end{array}$ & $\begin{array}{l}\text {-walking } \\
\text { speed } \\
\text {-strength of } \\
\text { the lower } \\
\text { limbs } \\
\text {-Endurance }\end{array}$ & $\begin{array}{l}\text {-walking } \\
\text { speed } \\
\text {-strength of } \\
\text { the lower } \\
\text { limbs } \\
\text {-balance } \\
\text { - Endurance }\end{array}$ & $\begin{array}{l}\text {-walking } \\
\text { speed } \\
\text { - strength } \\
\text { of the } \\
\text { lower } \\
\text { limbs } \\
\text { - balance } \\
\text { - Endurance }\end{array}$ & $\begin{array}{l}\text { Walking } \\
\text { speed } \\
\text { - strength of } \\
\text { the lower } \\
\text { limbs }\end{array}$ & $\begin{array}{l}\text { walking } \\
\text { speed } \\
\text {-strength of } \\
\text { the lower } \\
\text { limbs } \\
\text {-balance } \\
\text { Endurance }\end{array}$ & $\begin{array}{l}\text { walking } \\
\text { speed } \\
\text {-functional } \\
\text { ambulation }\end{array}$ \\
\hline & secondary & & Secondary & & secondary & Secondary: & & \\
\hline & Pain & & $\begin{array}{l}\text {-pain } \\
\text {-spasticity }\end{array}$ & & $\begin{array}{l}\text { muscle } \\
\text { tone }\end{array}$ & $\begin{array}{l}\text { Balance } \\
\text { Posture } \\
\text { control }\end{array}$ & & \\
\hline $\begin{array}{l}\text { Conclusio } \\
\text { ns }\end{array}$ & $\begin{array}{l}\text { RAGT can } \\
\text { improve } \\
\text { walking in } \\
\text { patients } \\
\text { with ISCI } \\
\text { but not } \\
\text { better than } \\
\text { lower } \\
\text { extremity } \\
\text { strength }\end{array}$ & $\begin{array}{l}\text { both manual } \\
\text { and } \\
\text { RAGT gave } \\
\text { small gains } \\
\text { among late- } \\
\text { onset ISCI } \\
\text { patients }\end{array}$ & $\begin{array}{l}\text { training with } \\
\text { the Lokomat } \\
\text { system } \\
\text { improve } \\
\text { walking } \\
\text { but not better } \\
\text { than those } \\
\text { produced by } \\
\text { a } \\
\text { conventional } \\
\text { overground } \\
\text { training } \\
\text {. }\end{array}$ & $\begin{array}{l}\text { Exoskeleton- } \\
\text { assisted gait } \\
\text { training for } \\
\text { individuals } \\
\text { with iSCI } \\
\text { could } \\
\text { improve gait } \\
\text { and gait } \\
\text { function }\end{array}$ & $\begin{array}{l}\text { Cable- } \\
\text { driven } \\
\text { robotic } \\
\text { resistance } \\
\text { training } \\
\text { may be } \\
\text { used as an } \\
\text { adjunct to } \\
\text { BWSTT } \\
\text { for } \\
\text { improving } \\
\text { overground } \\
\text { walking } \\
\text { Function in } \\
\text { incomplete } \\
\text { SCI } \\
\text { patients. }\end{array}$ & $\begin{array}{l}\text { BWSLT with } \\
\text { manual } \\
\text { assistance } \\
\text { was well } \\
\text { tolerated and } \\
\text { led to } \\
\text { statistically } \\
\text { non- } \\
\text { significant } \\
\text { improvement } \\
\text { s in walking } \\
\text { and lower } \\
\text { extremity } \\
\text { muscle } \\
\text { strength. }\end{array}$ & $\begin{array}{l}\text { Improve } \\
\text { walking } \\
\text { ability and } \\
\text { psychologica } \\
\text { l well-being } \\
\text { following a } \\
\text { concentrated } \\
\text { period of } \\
\text { ambulation } \\
\text { therapy, } \\
\text { regardless of } \\
\text { training } \\
\text { method. }\end{array}$ & $\begin{array}{l}\text { BWSTT in } \\
\text { comparison } \\
\text { with } \\
\text { traditional } \\
\text { exercise can } \\
\text { improve } \\
\text { more motor } \\
\text { function and } \\
\text { quality and } \\
\text { quantity of } \\
\text { walking in } \\
\text { people with } \\
\text { paraplegic } \\
\text { spinal cord } \\
\text { injury }\end{array}$ \\
\hline
\end{tabular}

\section{Meta-analysis:}

\section{1- Walking speed (10 MWT)}

Five studies assessed Ten Minute Walking Test (10 MWT) between the experimental group and control group to improve $10 \mathrm{MWT}$ with chronic incomplete spinal cord injury (Figure 1). There was considerable heterogeneity in 10 MWT between Five studies ( $\mathrm{n}=5$ studies, $\mathrm{n}=113$ participants, $\mathrm{P}<0.0001 ; I^{2}=86 \%$ ). There was no significant difference $(\mathrm{P}=0.97 ; \mathrm{P}>0.05)$ in overall effect of $10 \mathrm{MWT}(\mathrm{SMD}=0.02 ; 95 \% \mathrm{CI},-1.31$ to 1.36$)$ between experimental group and control group. Sensitivity analysis showed that 10 MWT was significant except by excluding one trial at a time from pooled effects to determine whether any one study was particularly influential. A significant overall effect between the experimental group and control group in $10 \mathrm{MWT}$ (sensitivity 5; $\mathrm{SMD}=0.63 ; 95 \% \mathrm{CI}, 0.21$ to $1.05 ; \mathrm{P}=0.003, I^{2}=0 \%$ ) was observed after removal one study Piira et al. ${ }^{(22)}$ according to sensitivity analysis matrix. 
https://ejhm.journals.ekb.eg/

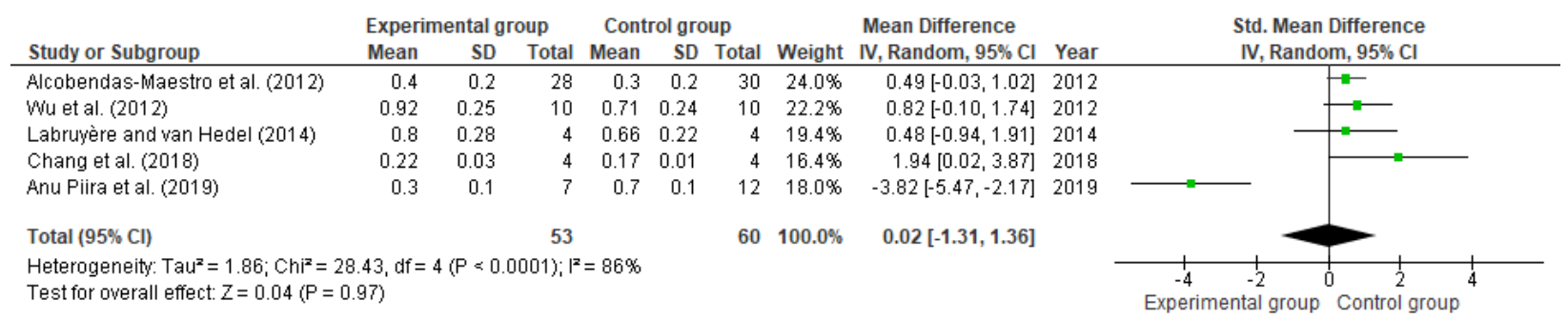

\section{Sensitivity (1)}

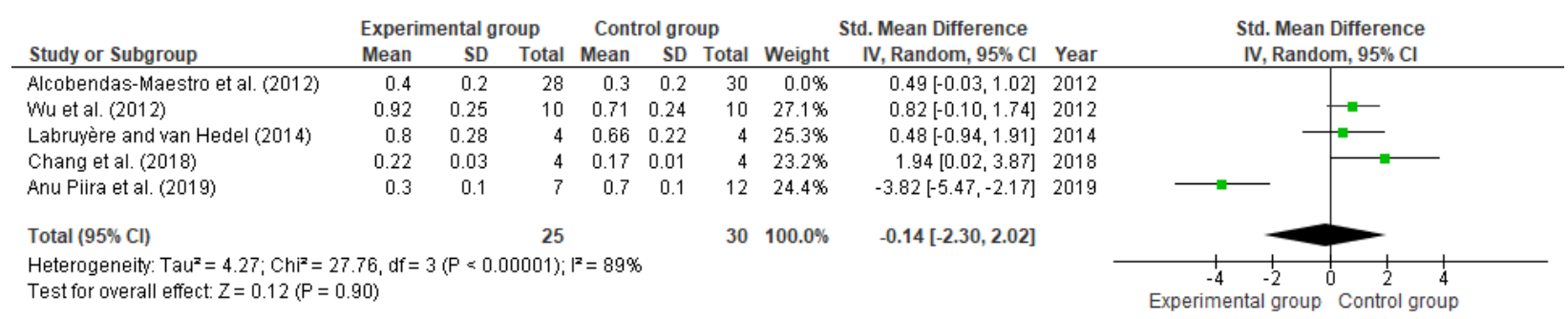

\section{Sensitivity (2)}

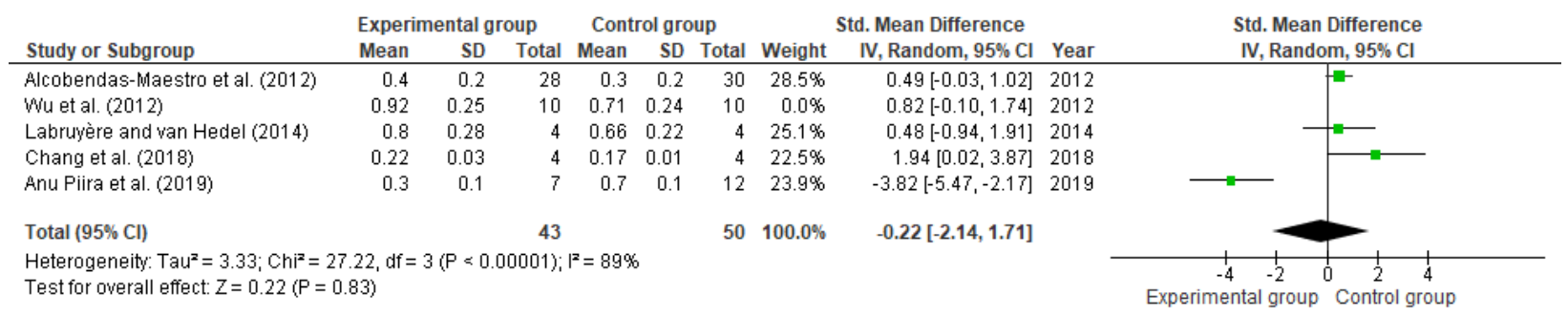

\section{Sensitivity (3)}

\begin{tabular}{|c|c|c|c|c|c|c|c|c|c|c|}
\hline \multirow[b]{2}{*}{ Study or Subgroup } & \multicolumn{3}{|c|}{ Experimental group } & \multicolumn{3}{|c|}{ Control group } & \multicolumn{3}{|c|}{ Std. Mean Difference } & \multirow{2}{*}{$\begin{array}{l}\text { Std. Mean Difference } \\
\text { IV, Random, } 95 \% \mathrm{Cl}\end{array}$} \\
\hline & Mean & SD & Total & Mean & SD & Total & Weight & IV, Random, $95 \% \mathrm{Cl}$ & Year & \\
\hline Alcobendas-Maestro et al. (2012) & 0.4 & 0.2 & 28 & 0.3 & 0.2 & 30 & $28.8 \%$ & $0.49[-0.03,1.02]$ & 2012 & - \\
\hline Wu et al. (2012) & 0.92 & 0.25 & 10 & 0.71 & 0.24 & 10 & $27.1 \%$ & $0.82[-0.10,1.74]$ & 2012 & \\
\hline Labruyère and van Hedel (2014) & 0.8 & 0.28 & 4 & 0.66 & 0.22 & 4 & $0.0 \%$ & $0.48[-0.94,1.91]$ & 2014 & \\
\hline Chang et al. (2018) & 0.22 & 0.03 & 4 & 0.17 & 0.01 & 4 & $21.2 \%$ & $1.94[0.02,3.87]$ & 2018 & \\
\hline Anu Piira et al. (2019) & 0.3 & 0.1 & 7 & 0.7 & 0.1 & 12 & $22.9 \%$ & $-3.82[-5.47,-2.17]$ & 2019 & \\
\hline Total $(95 \% \mathrm{Cl})$ & & & 49 & & & 56 & $100.0 \%$ & $-0.10[-1.76,1.56]$ & & \\
\hline \multicolumn{10}{|c|}{$\begin{array}{l}\text { Heterogeneity: } \operatorname{Tau}^{2}=2.42 ; \mathrm{Chi}^{2}=28.39, \mathrm{df}=3(\mathrm{P}<0.00001) ; \mathrm{I}^{2}=89 \% \\
\text { Test for overall effect: } Z=0.12(\mathrm{P}=0.91)\end{array}$} & $\begin{array}{cccc}-4 & -2 & 0 & 2 \\
\text { Experimental group } & \text { Control g }\end{array}$ \\
\hline
\end{tabular}

\section{Sensitivity (4)}

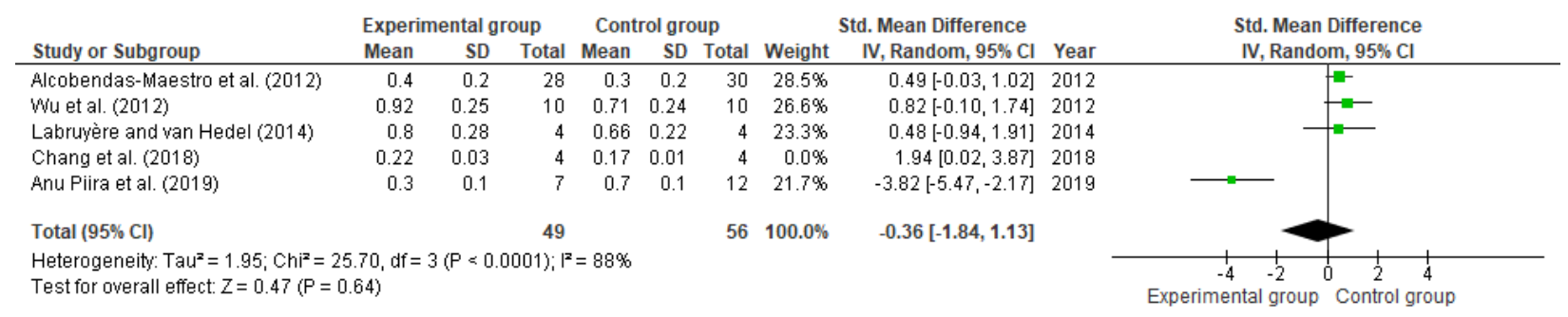

\section{Sensitivity (5)}

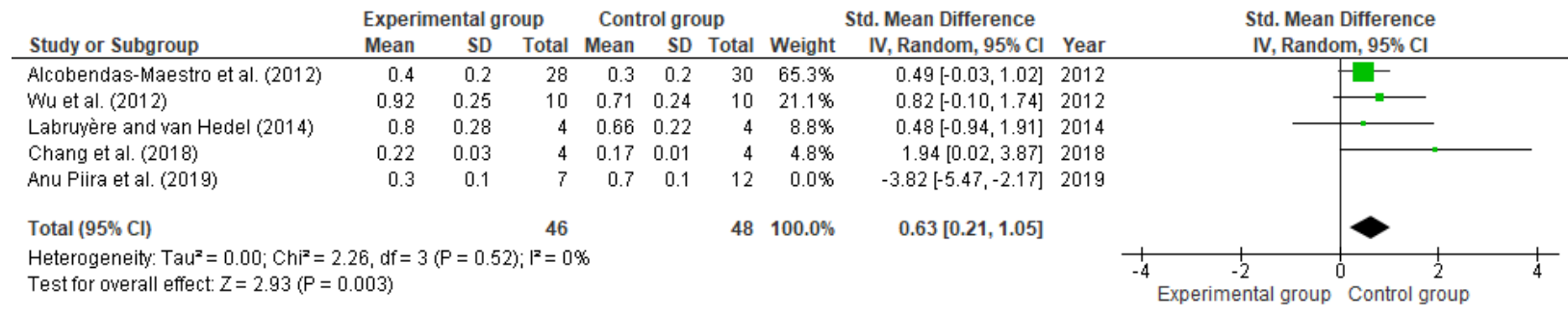

Figure (1): Standardized mean differences (95\% CI) of ten-minute walking speed (10 MWT) after robotic-assisted as compared with control from five studies. Sensitivity analysis showed that 10 MWT was significant except by excluding one trial Piira $\boldsymbol{e t}$ al. ${ }^{(26)}$ at a time from pooled effects to determine whether anyone study was particularly influential. Susceptibility analysis showed that there was stability of results for this meta-analysis 


\section{2- Endurance (6 MWT)}

Four studies assessed the Six Meter Walking Test (6 MWT) between the experimental group and control group to improve 6 MWT with chronic incomplete spinal cord injury (Figure 2). There was considerable heterogeneity in 6 MWT between four studies ( $\mathrm{n}=4$ studies, $\mathrm{n}=105$ participants, $\mathrm{P}<0.00001 ; I^{2}=90 \%$ ). There was no significant difference $(\mathrm{P}=0.97 ; \mathrm{P}>0.05)$ in overall effect of $6 \mathrm{MWT}(\mathrm{SMD}=-0.07 ; 95 \% \mathrm{CI},-1.59$ to 1.44$)$ between experimental group and control group. Sensitivity analysis showed that 6 MWT was significant except by excluding one trial at a time from pooled effects to determine whether any one study was particularly influential. A significant overall effect between the experimental and control groups in $6 \mathrm{MWT}$ (sensitivity 4; $\mathrm{SMD}=0.82 ; 95 \% \mathrm{CI}, 0.12$ to $1.51 ; \mathrm{P}=0.02, I^{2}=45 \%$ ) was observed after removal one study Piira $\boldsymbol{e t}$ al. ${ }^{(22)}$ according to sensitivity analysis matrix.

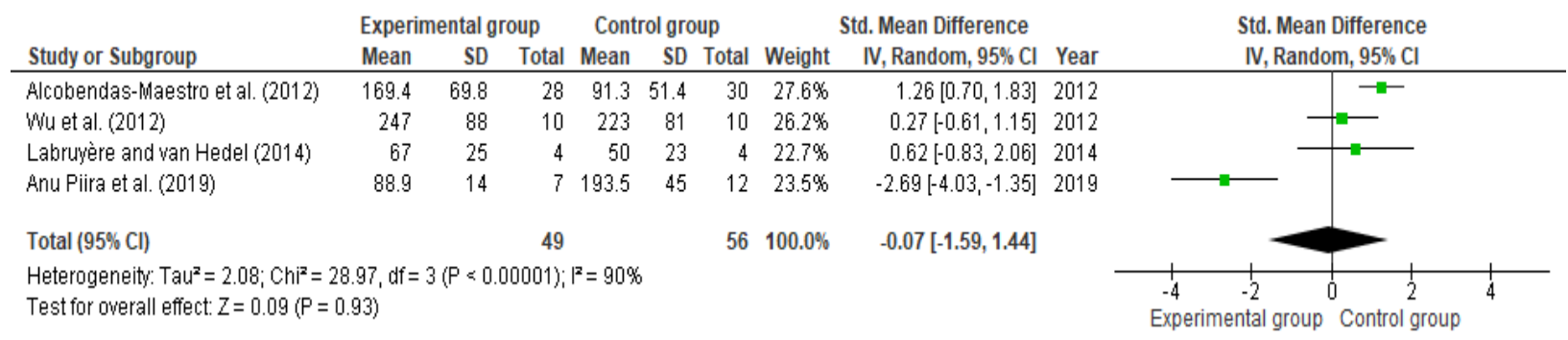

Sensitivity (1)

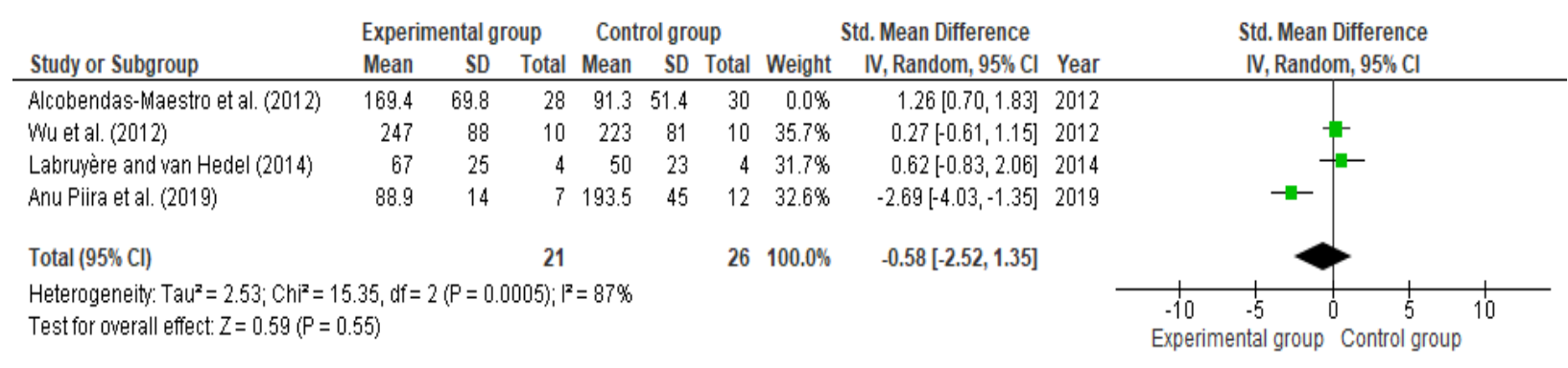

Sensitivity (2)

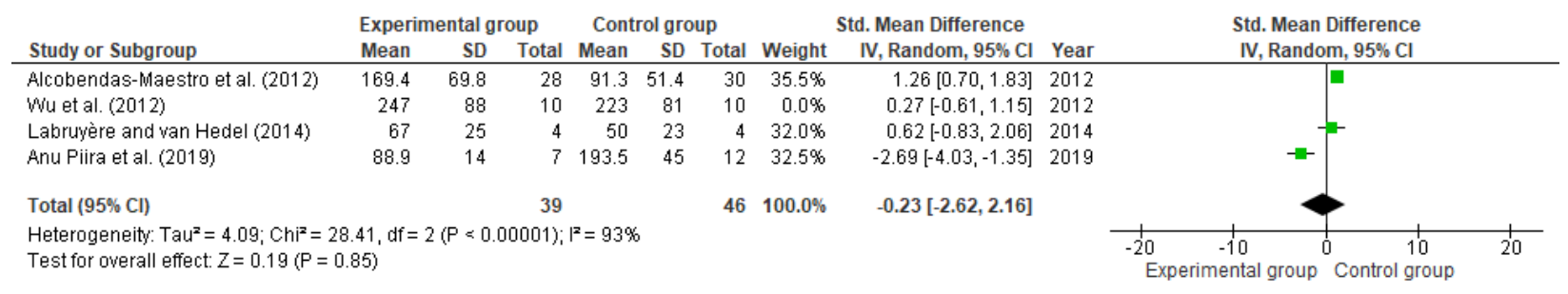

\section{Sensitivity (3)}

\begin{tabular}{|c|c|c|c|c|c|c|c|c|c|c|}
\hline \multirow[b]{2}{*}{ Study or Subgroup } & \multicolumn{3}{|c|}{ Experimental group } & \multicolumn{3}{|c|}{ Control group } & \multicolumn{3}{|c|}{ Std. Mean Difference } & \multirow{2}{*}{$\begin{array}{l}\text { Std. Mean Difference } \\
\text { IV, Random, } 95 \% \mathrm{Cl}\end{array}$} \\
\hline & Mean & SD & Total & Mean & SD & Total & Weight & IV, Random, $95 \% \mathrm{Cl}$ & Year & \\
\hline Alcobendas-Maestro et al. (2012) & 169.4 & 69.8 & 28 & 91.3 & 51.4 & 30 & $35.2 \%$ & $1.26[0.70,1.83]$ & 2012 & 를 \\
\hline Wu et al. (2012) & 247 & 88 & 10 & 223 & 81 & 10 & $33.8 \%$ & $0.27[-0.61,1.15]$ & 2012 & + \\
\hline Labruyère and van Hedel (2014) & 67 & 25 & 4 & 50 & 23 & 4 & $0.0 \%$ & $0.62[-0.83,2.06]$ & 2014 & \\
\hline Anu Piira et al. (2019) & 88.9 & 14 & 7 & 193.5 & 45 & 12 & $31.0 \%$ & $-2.69[-4.03,-1.35]$ & 2019 & $\rightarrow-$ \\
\hline Total $(95 \% \mathrm{Cl})$ & & & 45 & & & 52 & $100.0 \%$ & $-0.30[-2.24,1.64]$ & & \\
\hline \multicolumn{11}{|c|}{ 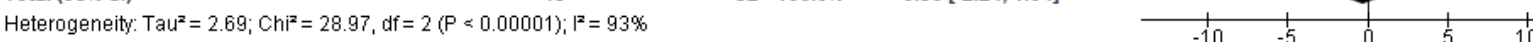 } \\
\hline
\end{tabular}

\section{Sensitivity (4)}

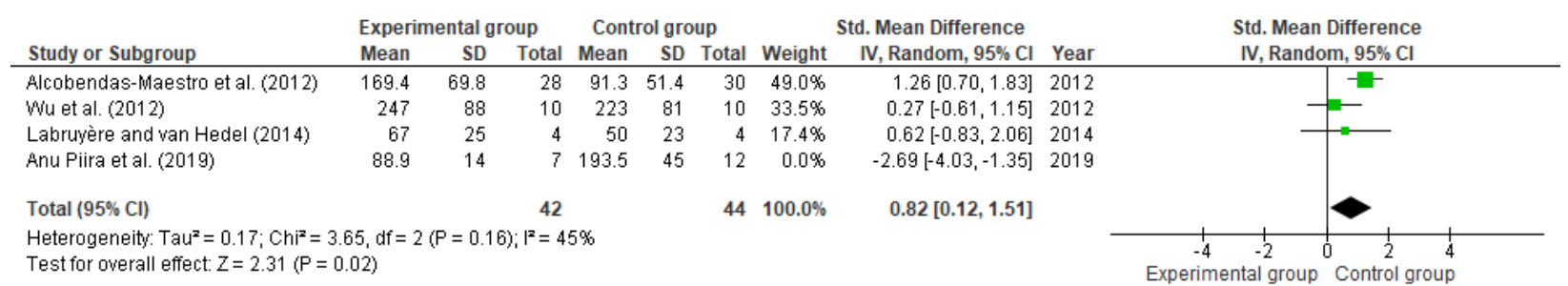

Figure (2): Standardized mean differences (95\% CI) of the six-meter walking test (6 MWT) after robotic-assisted as compared with a control from five studies. Sensitivity analysis showed that 6 MWT was significant except by excluding one trial Piira et al. ${ }^{(26)}$ at a time from pooled effects to determine whether any one study was particularly influential. Susceptibility analysis showed that there was stability of results for this meta-analysis 


\section{3- Strength of lower limbs (LEMS)}

Three studies assessed Lower Extremity Motor Score (LEMS) between the experimental and control groups to improve LEMS with chronic incomplete spinal cord injury (Figure 3). There was no heterogeneity in LEMS between three studies ( $\mathrm{n}=3$ studies, $\mathrm{n}=85$ participants, $\left.\mathrm{P}=0.62 ; I^{2}=0 \%\right)$. There was no significant difference $(\mathrm{P}=0.24 ; \mathrm{P}>0.05)$ in overall effect of LEMS ( $\mathrm{SMD}=0.26$; $95 \% \mathrm{CI},-0.17$ to 0.69 ) between experimental group and control group. Sensitivity analysis showed that LEMS was not significant $(\mathrm{P}>0.05)$ by excluding one trial at a time from pooled effects to determine LEMS after removal of any study throughout sensitivity analysis matrix.

\begin{tabular}{|c|c|c|c|c|c|c|c|c|c|c|}
\hline \multirow[b]{2}{*}{ Study or Subgroup } & \multicolumn{3}{|c|}{ Experimental group } & \multicolumn{3}{|c|}{ Control group } & \multicolumn{3}{|c|}{ Std. Mean Difference } & \multirow{2}{*}{$\begin{array}{l}\text { Std. Mean Difference } \\
\text { IV, Random, } 95 \% \mathrm{Cl}\end{array}$} \\
\hline & Mean & SD & Total & Mean & SD & Total & Weight & IV, Random, $95 \% \mathrm{Cl}$ & Year & \\
\hline Alcobendas-Maestro et al. (2012) & 40 & 35 & 28 & 35 & 29.7 & 30 & $70.2 \%$ & $0.15[-0.36,0.67]$ & 2012 & 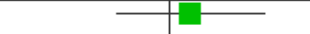 \\
\hline Labruyère and van Hedel (2014) & 41.4 & 6.9 & 4 & 40.4 & 6.6 & 4 & $9.7 \%$ & $0.13[-1.26,1.52]$ & 2014 & \\
\hline Anu Piira et al. (2019) & 33.8 & 1 & 7 & 27.4 & 11 & 12 & $20.1 \%$ & $0.69[-0.27,1.65]$ & 2019 & \\
\hline Total $(95 \% \mathrm{Cl})$ & & & 39 & & & 46 & $100.0 \%$ & $0.26[-0.17,0.69]$ & & \\
\hline \multicolumn{11}{|c|}{ 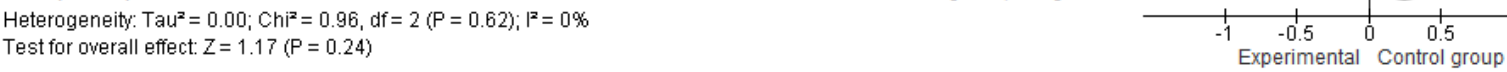 } \\
\hline
\end{tabular}

\section{Sensitivity (1)}

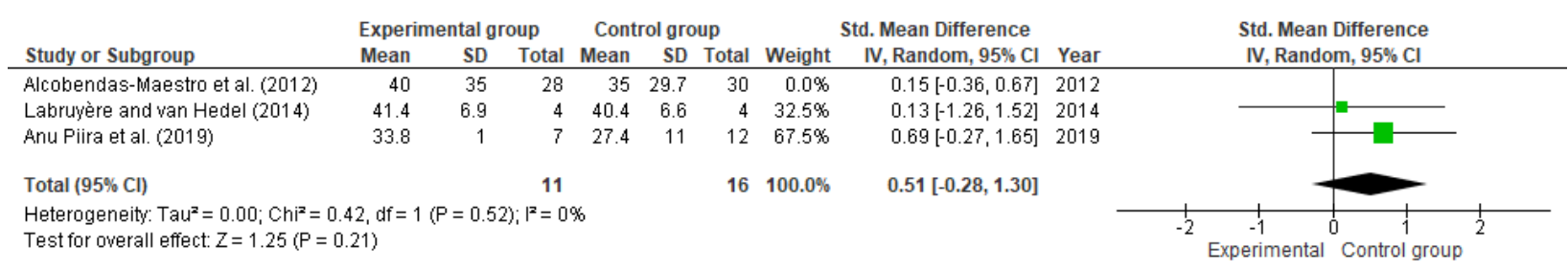

\section{Sensitivity (2)}

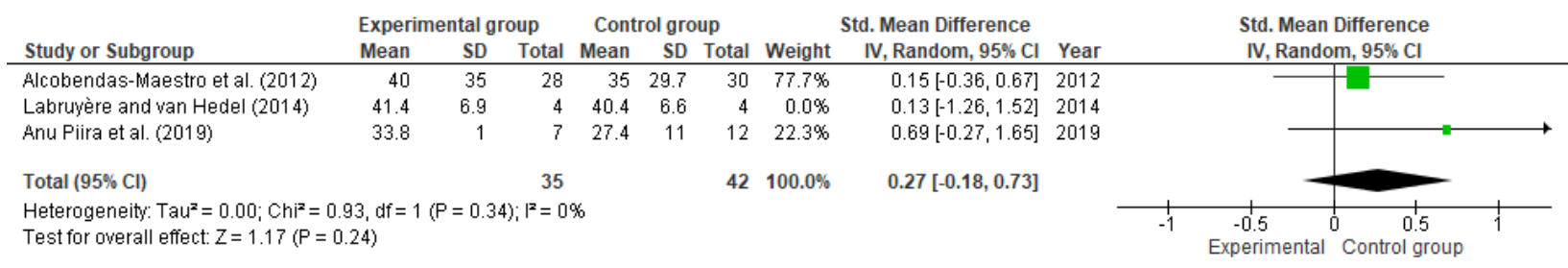

\section{Sensitivity (3)}

\begin{tabular}{|c|c|c|c|c|c|c|c|c|c|c|}
\hline \multirow[b]{2}{*}{ Study or Subgroup } & \multicolumn{3}{|c|}{ Experimental group } & \multicolumn{3}{|c|}{ Control group } & \multicolumn{3}{|c|}{ Std. Mean Difference } & \multirow{2}{*}{$\begin{array}{c}\text { Std. Mean Difference } \\
\text { IV, Random, } 95 \% \mathrm{Cl}\end{array}$} \\
\hline & Mean & SD & Total & Mean & SD & Total & Weight & IV, Random, $95 \% \mathrm{Cl}$ & Year & \\
\hline Alcobendas-Maestro et al. (2012) & 40 & 35 & 28 & 35 & 29.7 & 30 & $87.9 \%$ & $0.15[-0.36,0.67]$ & 2012 & \begin{tabular}{l|l}
-1 \\
\end{tabular} \\
\hline Labruyère and van Hedel (2014) & 41.4 & 6.9 & 4 & 40.4 & 6.6 & 4 & $12.1 \%$ & $0.13[-1.26,1.52]$ & 2014 & \\
\hline Anu Piira et al. (2019) & 33.8 & 1 & 7 & 27.4 & 11 & 12 & $0.0 \%$ & $0.69[-0.27,1.65]$ & 2019 & \\
\hline Total $(95 \% \mathrm{Cl})$ & & & 32 & & & 34 & $100.0 \%$ & $0.15[-0.33,0.63]$ & & \\
\hline \multicolumn{10}{|c|}{$\begin{array}{l}\text { Heterogeneity: } \operatorname{Tau}^{2}=0.00 ; \mathrm{Chi}^{2}=0.00, \mathrm{df}=1(P=0.98) ; \mathrm{I}^{2}=0 \% \\
\text { Test for overall effect: } Z=0.61(P=0.54)\end{array}$} & $\begin{array}{cccc}1 & 1 & 1 & 1 \\
-1 & -0.5 & 0 & 0.5 \\
& \text { Experimental } & & \\
& \text { Control group }\end{array}$ \\
\hline
\end{tabular}

Figure (3): Standardized mean differences (95\% CI) of lower extremity motor score (LEMS) after robotic-assisted as compared with a control from five studies. Sensitivity analysis showed that LEMS was not significant by excluding one trial at a time from pooled effects to determine whether any one study was particularly influential. Susceptibility analysis showed that there was stability of results for this meta-analysis

\section{4- Berg balance scale (BBS)}

Four studies assessed Berg Balance Scale (BBS) between the experimental and control groups to improve BBS with chronic incomplete spinal cord injury (Figure 4). There was no heterogeneity in BBS between four studies $(n=4$ studies, $\mathrm{n}=47$ participants, $\left.\mathrm{P}=0.89 ; I^{2}=0 \%\right)$. There was no significant difference $(\mathrm{P}=0.97 ; \mathrm{P}>0.05)$ in overall effect of BBS ( $\mathrm{SMD}=0.11 ; 95 \% \mathrm{CI},-0.47$ to 0.69 ) between experimental group and control group. Sensitivity analysis showed that BBS was not significant $(\mathrm{P}>0.05)$ by excluding one trial at a time from pooled effects to determine BBS after removal of any study throughout sensitivity analysis matrix. 
https://ejhm.journals.ekb.eg/

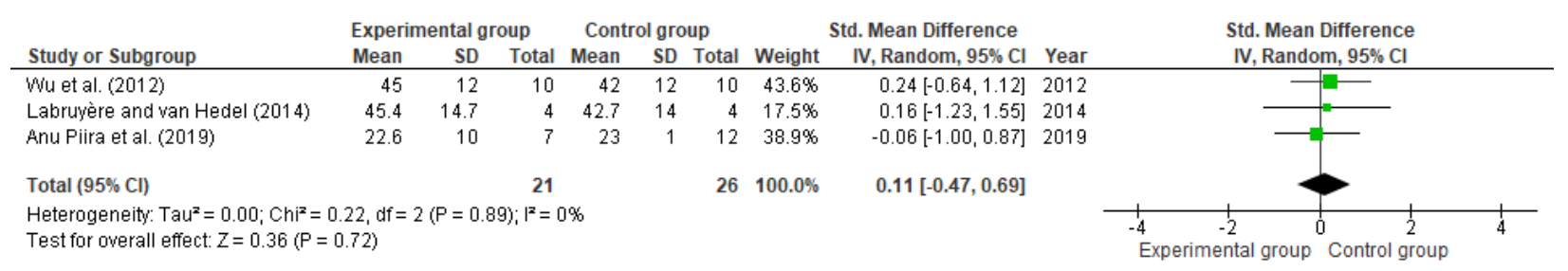

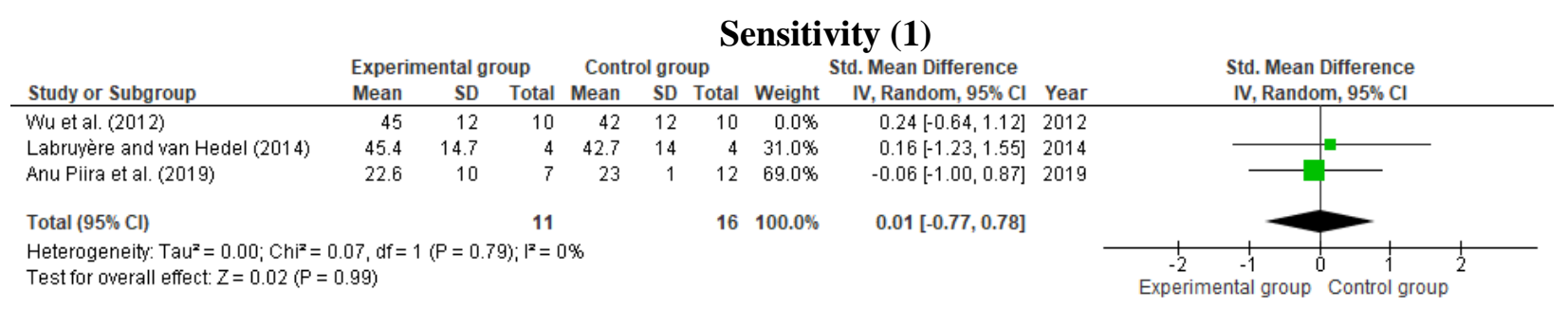

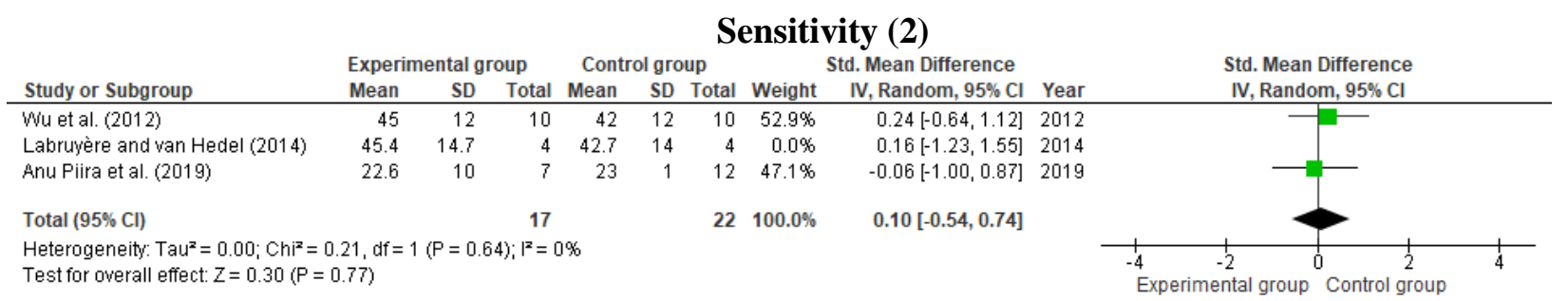

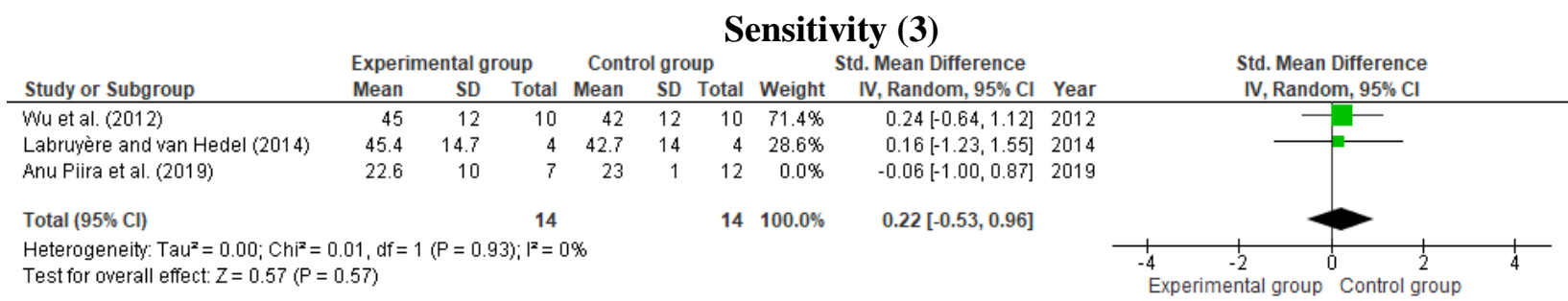

Figure (4): Standardized mean differences (95\% CI) of Berg balance scale (BBS) after robotic-assisted as compared with a control from five studies. Sensitivity analysis showed that BBS was not significant by excluding one trial at a time from pooled effects to determine whether any one study was particularly influential. Susceptibility analysis showed that there was stability of results for this meta-analysis.

\section{DISCUSSION}

The purpose of the current systematic review was to evaluate the Effectiveness of Assistive Devices on Gait in Patients with incomplete Spinal Cord Injury. This review includes studies published from January 2010 to 2021 which resulted in only eight RCTs that have undergone the systematic review. The review revealed good quality evidence to support usage of Assistive Devices on Gait in Patients with incomplete Spinal Cord Injury as the PEDRo scores of the reviewed studies were between 4 and 9 . The primary objective of using assistive devices is to induce several different neurophysiological mechanisms to restore walking ability, including the activation of central pattern generators, task-specific stepping practice, and massed exercise.

Various locomotor training methods have been used in attempts to recover walking function after incomplete spinal cord injury (ISCI) robot-assisted locomotor training (RALT) had effects similar to other types of body weight supported locomotor training, and to the same amount of conventional training or physical therapy, in re-establishing walking independence and endurance/ distance walked. The articles included in this review are characterized by providing results that help in determining our study aimed to assess the effects of assistive devices on improvement in walking-related functional outcomes in patients with incomplete SCI.
The present systematic review analyzed eight RCTs by applying strict selection criteria for inclusion. Meta-analysis was applied for five studies including Labruyère et al. ${ }^{(21)}$, Piira et al. ${ }^{(22)}$, Alcobendas et al. (23), Chang et al. ${ }^{(24)}$, and Wu et al. (25), and a descriptive analysis was applied for three studies including Piira $\boldsymbol{e t}$ al. ${ }^{(26)}$, Alexeeva et al. ${ }^{(27)}$ and Sadeghi et al. ${ }^{(28)}$. The metaanalysis performed in this review revealed a statistically no significant effect of Assistive Devices on Gait in Patients with incomplete Spinal Cord Injury. And there are no RCTs enough to investigate robotic devices that have a significant role in our case study.

five studies show if the assistive devices play a significant role in gait in patients with incomplete spinal cord injury. Alcobendas et al. (23) investigate that Robotic-assisted training was equivalent to conventional walk training in patients with a variety of non-progressive spinal cord pathologies for walking speed, but the need for orthotics and assistive devices was reduced, and this may be due to greater leg strength in the robotic group. We found Labruyère $\boldsymbol{e t}$ al. ${ }^{(21)}$ agree with the result of this paper and he said that there were no significant differences in changes in scores between the 2 interventions, but in cases with chronic iSCI dependent on walking assistance, RAGT was not more effective in improving walking-related outcome compared to lower extremity strength training. 
Some studies showed that robotic devices have a significant role on gait that Wu et al. ${ }^{(25)}$ said "Cabledriven robotic resistance training may be used as an adjunct to BWSTT for improving over-ground walking function in humans with incomplete SCI, particularly for those patients with the relatively high function", and Chang et al. ${ }^{(24)}$ proved that Exoskeleton-assisted gait training for individuals with iSCI could improve gait and gait function. But we found that Piira et al. ${ }^{\text {(22) }}$ disagreed with this result and he said that Late-onset robot-assisted locomotor training did not improve independent walking function, and the non-significant effect was seen on muscle strength and balance.

\section{CONCLUSION}

According to investigated papers, robotic devices have a significant effect on gait in patients with incomplete spinal cord injury but our systematic review evidenced that robotic devices have no significant effect on Gait in patients with incomplete spinal cord injury in comparison with other devices but have a good effect as others. As all assistive devices have a good role in improving gait in patients with iSCI We need more clinical studies to investigate the effect of robotic devices on gait to have a more useful systemic review.

\section{Financial support and sponsorship: Nil. \\ Conflict of interest: Nil.}

\section{REFERENCES}

1. Alizadeh A, Dyck S, Karimi-Abdolrezaee $S$ (2019): Traumatic spinal cord injury: an overview of pathophysiology, models and acute injury mechanisms. Frontiers in Neurology, 10:282-87.

2. Jefferson R, Cadotte D, Fehlings M (2012): Clinical predictors of neurological outcome, functional status, and survival after traumatic spinal cord injury: a systematic review. Journal of Neurosurgery: Spine, 17: 11-26.

3. Gittler M, McKinley W, Stiens S et al. (2002): Spinal cord injury medicine. 3. Rehabilitation outcomes. Arch Phys Med Rehabil., 83: $65-71$

4. Atrice M, Morrison S, McDowell S et al. (2006): Traumatic spinal cord injury. In: Umphred DA. Neurological Rehabilitation. 5th ed. St. Louis, MO: Mosby. Pp. 605-657.

5. Sisto S, Druin E, MachtSliwinski M (2008): Spinal Cord Injuries: Management and Rehabilitation. St. Louis, MO: Mosby. Pp. 512533.

6. van Hedel H, Dietz V (2010): Rehabilitation of locomotion after spinal cord injury. Restor Neurol Neuro-Sci., 28:123-134.

7. Harkema S, Schmidt-Read M, Lorenz D et al. (2011): Balance and ambulation improvements in individuals with chronic incomplete spinal cord injury using locomotor training-based rehabilitation. Arch Phys Med Rehabil., 93: 919-21.

8. Kawamoto H, Sankai Y (2005): Assist method based on Phase Sequence and muscle force condition for HAL. Adv Robot., 19(7):714-734

9. Donati A, Shokur S, Morya E et al. (2016): Long-Term Training with a Brain-Machine Interface-Based Gait Protocol Induces Partial Neurological Recovery in Paraplegic Patients. Sci Rep., 6: 1-16.

10. Field-Fote E, Yang J, Basso D et al. (2016): Supra spinal control predicts locomotor function and forecasts responsiveness to training after spinal cord injury. J Neurotrauma., 34(9):1813-1825.

11. Hornby T, Zemon D, Campbell T (2005): Robotic-assisted, bodyweight supported treadmill training in individuals following motor incomplete spinal cord injury. Phys Ther., 85(1):52-66.

12. Wirz M, Bastiaenen C, de Bie R et al. (2011): Effectiveness of automated locomotor training in patients with acute incomplete spinal cord injury: a randomized controlled multicenter trial. BMC Neurol., 11(1):60.

13. Wirz M, Zemon D, Rupp R et al. (2005): Effectiveness of automated locomotor training in patients with chronic incomplete spinal cord injury: A multicenter trial. Arch Phys Med Rehabil., 86:672-77.

14. Effing T, van Meeteren N, van Asbeck $F$ et al. (2006): Body weight-supported treadmill training in chronic incomplete spinal cord injury: a pilot study evaluating functional health status and quality of life. Spinal Cord, 44:287-91.

15. Liberson W, Holmquest H, Scot $D$ et al. (1961): Functional electrotherapy: stimulation of the peroneal nerve synchronized with the swing phase of the gait of hemiplegic patients. Arch Phys Med Rehabil., 42:101-105.

16. O'sullivan S, Schmitz T, Fulk G (2014): Physical Rehabilitation. (6th ed.): Philadelphia: FA Davis Company. Pp. 108-109.

(S(oyulxb452alnt1aej1nfow45))/

https://www.scirp.org/ reference/ReferencesPapers.aspx?ReferenceID=1952416

17. Gopalakrishnan S, Ganeshkumar P (2013): Systematic Reviews and Meta-analysis: Understanding the Best Evidence in Primary Healthcare. J Family Med Prim Care, 2(1): 9-14.

18. Haynes R, Deveaux P, Guyatt G (2002): Physicians and patients" choices in evidence-evidence-based practice. British Medical Journal, 324: 1350-55.

19. Moher D, Liberati A, Tetzlaff $\mathrm{J}$ et al. (2009): Preferred reporting items for systematic reviews and meta-analyses: the PRISMA statement. PLoS Medicine, 6(7): 97-103.

20. Maher C, Sherrington C, Elkins M et al. (2004): Challenges for evidence-based physical therapy: accessing and interpreting highquality evidence on therapy. Physical Therapy, 84(7): 644-654.

21. Labruyère $R$, van Hedel $H$ (2014): Strength training versus robot-assisted gait training after incomplete spinal cord injury: a randomized pilot study in patients depending on walking assistance. Journal of Neuroengineering and Rehabilitation, 11(1): 1-12.

22. Piira A, Lannem A, Sørensen M et al. (2019): Robot-assisted locomotor training did not improve walking function in patients with chronic incomplete spinal cord injury: a randomized clinical trial. Journal of Rehabilitation Medicine, 51(5): 385-389.

23. Alcobendas-Maestro M, Esclarín-Ruz A, Casado-López R et al. (2012): Lokomat robotic-assisted versus overground training within 3 to 6 months of incomplete spinal cord lesion: randomized controlled trial. Neurorehabilitation and Neural Repair, 26(9): 1058-1063.

24. Chang S, Afzal T, Berliner J et al. (2018): Exoskeleton-assisted gait training to improve gait in individuals with spinal cord injury: a pilot randomized study. Pilot and Feasibility Studies, 4(1): 1-10.

25. Wu M, Landry J, Schmit B et al. (2012): Robotic resistance treadmill training improves locomotor function in human spinal cord injury: a pilot study. Archives of Physical Medicine and Rehabilitation, 93(5): 782-789.

26. Piira A, Lannem A, Sørensen M et al. (2019): Manually assisted body-weight supported locomotor training does not re-establish walking in non-walking subjects with chronic incomplete spinal cord injury: A randomized clinical trial. Journal of Rehabilitation Medicine, 51:113-9.

27. Alexeeva N, Sames C, Jacobs P et al. (2011): Comparison of training methods to improve walking in persons with chronic spinal cord injury: a randomized clinical trial. The Journal of Spinal cord Medicine, 34(4): 362-379.

28. Sadeghi H, Banitalebi E, Raeisi D (2015): The effect of bodyweight-supported training exercises on functional ambulation profile in patients with paraplegic spinal cord injury. Physical Treatments-Specific Physical Therapy Journal, 4(4): 205-212.

29. Maher $C$, Sherrington $C$, Herbert $R$ et al. (2003): Reliability of the PEDro scale for rating quality of randomized controlled trials. Phys Ther., 83: 713-21.

30. Higgins J, Altman D, Gøtzsche $P$ et al. (2011): The Cochrane Collaboration's tool for assessing the risk of bias in randomized trials. BMJ., 343: 5928-35. 